\title{
Combination therapy of tyrosine kinase receptor inhibitor TSU-68 (SU6668) and paclitaxel inhibits subcutaneous xenografts of endometrial cancer
}

\author{
SHIZUO MACHIDA, YASUSHI SAGA, YUJI TAKEI, KAYOKO TAKAHASHI, HIROAKI NONAKA, \\ HIROYUKI FUJIWARA, MICHITAKA OHWADA and MITSUAKI SUZUKI
}

Department of Obstetrics and Gynecology, Jichi Medical University, Tochigi 329-0498, Japan

Received June 4, 2008; Accepted September 8, 2008

DOI: $10.3892 / \mathrm{mmr}+00000038$

\begin{abstract}
TSU-68 is a small-molecular-weight synthetic inhibitor of the tyrosine kinase receptors Flk-1/KDR, PDGFRß and FGFR1, which are involved in angiogenesis. Using a mouse model in which endometrial cancer was subcutaneously implanted, we investigated the effects of TSU-68 alone or in combination with paclitaxel. We subcutaneously implanted a cell strain of endometrial cancer, HEC1A, into BALB/c nude mice. TSU-68 was orally administered every day, while paclitaxel was intraperitoneally injected once a week, and the rates of subcutaneous tumor proliferation were compared. In a group treated with high-dose (200 mg/kg/day) TSU-68 alone, subcutaneous tumor proliferation was significantly inhibited in comparison with a vehicle-treated control group $(\mathrm{p}<0.05)$. In groups treated with low-dose TSU-68 or paclitaxel alone (100 and $10 \mathrm{mg} / \mathrm{kg} / \mathrm{day}$, respectively), tumor proliferation was not significantly inhibited. In a low-dose combination therapy group $(100 \mathrm{mg} / \mathrm{kg} /$ day of TSU-68 $+10 \mathrm{mg} / \mathrm{kg} /$ day of paclitaxel), tumor proliferation was significantly inhibited in comparison with the control and low-dose TSU-68 or paclitaxel therapy groups $(\mathrm{p}<0.01)$. High-dose monotherapy with TSU-68 inhibited the proliferation of the subcutaneously implanted tumor. Furthermore, a combination of TSU-68 and paclitaxel at a low dose, one at which respective monotherapy was not effective, inhibited tumor proliferation. Combination therapy with the two agents may therefore be useful for treating endometrial cancer.
\end{abstract}

\section{Introduction}

In the United States, endometrial cancer is the fourth most common disease among malignant tumors in women. The

Correspondence to: Dr Mitsuaki Suzuki, Department of Obstetrics and Gynecology, Jichi Medical School, Yakushiji, Shimotsuke, Tochigi 329-0498, Japan

E-mail: smachi@jichi.ac.jp

Key words: TSU-68, paclitaxel, endometrial cancer, angiogenesis, HEC1A annual number of patients newly developing this cancer is approximately 40,000 (1). In most patients, endometrial cancer is detected at an early stage, when the cancer is localized in the uterus. As, in such cases, radical surgery is possible, the 5 -year survival rate exceeds $80 \%$ (2). However, in advanced endometrial cancer patients with a FIGO stage of III/IV, which reflects extrauterine invasion, the 5-year survival rate remains low (0-40\%) $(2,3)$. In such patients, radiotherapy and combination chemotherapy have recently been applied in addition to surgery. However, the results of these are not satisfactory, and consequently the outcome of endometrial cancer treatment has not improved over the past 30 years (1). A new therapeutic strategy for the treatment of advanced endometrial cancer should therefore be established.

The proliferation and invasion of malignant tumors depend on angiogenesis (4). A recent study suggested the clinical usefulness of angiogenesis-inhibiting therapy for cancer, increasing its importance (5). Factors involved in tumor angiogenesis include vascular endothelial growth factor (VEGF), platelet-derived growth factor (PDGF) and basic fibroblast growth factor (bFGF) (6-9). In particular, VEGF plays a primary role (6). In patients with endometrial cancer, there was also a correlation between VEGF and angiogenesis (10), and VEGF has been reported to be a factor influencing the prognosis of endometrial cancer patients (10-12). This suggests the usefulness of angiogenesis-inhibiting therapy for endometrial cancer.

TSU-68, \{SU6668:(Z)-5-[(1,2-dihydro-2-oxo-3H-indol3-ylidene)methyl]-2,4-dimethyl-1H-pyrrole-3-ropanoic acid\}, is a small-molecular-weight synthetic kinase inhibitor of fetal liver kinase-1/kinase insert domain-containing receptor (Flk-1/ KDR), PDGF receptor $\beta$ (PDGFRß), and FGF receptor 1 (FGFR1), for VEGF, PDGF and bFGF, respectively $(13,14)$. It binds to the adenosine triphosphate-binding pocket of these three tyrosine kinase receptors and competitively inhibits their phosphorylation, thereby exhibiting inhibitory effects on vascular endothelial cell growth $(13,14)$. Several studies using various models in which solid cancer was subcutaneously implanted have confirmed that TSU-68 inhibits tumor angiogenesis and growth. Furthermore, this agent has been reported to induce apoptosis in vascular endothelial and tumor cells $(14,15)$.

In this study, we examined whether TSU-68 inhibits tumor proliferation in a subcutaneously endometrial cancer-implanted 
model for the purpose of its clinical application in patients with endometrial cancer. In addition, we investigated the effects of combination therapy with TSU-68 and paclitaxel, a conventional anticancer agent that has been reported to be effective for advanced endometrial cancer $(16,17)$.

\section{Materials and methods}

Cell line. A human endometrial endometrioid adenocarcinoma cell line, HEC-1A, was provided by Dr Kuramoto (Kitasato University, Japan).

Animals. We purchased 6-week-old female BALB/c nude mice from Clea Laboratories (Tokyo, Japan). The mice were acclimated under a pathogen-free environment. All animal experiments were performed according to the guidelines established by Jichi Medical University.

Drug administration. TSU-68 (SU6668) was provided by Taiho Pharmaceutical Co., Ltd. (Tokyo, Japan). We subcutaneously inoculated $6 \times 10^{6}$ cells/body of HEC-1A into BALB/c nude mice. Drug administration was initiated two weeks after inoculation. TSU-68 was dissolved in a carboxymethylcellulose vehicle, and orally administered 6 times a week. Paclitaxel, supplied by Bristol-Myers Squibb Co. (Tokyo, Japan), was dissolved in 50\% cremophor EL and 50\% dehydrated alcohol, then diluted with physiological saline. The solution was intraperitoneally injected once a week.

For monotherapy, high-dose TSU-68 (200 mg/kg/day) was orally administered every day. In the control group, a vehicle was administered.

For combination therapy, the mice were divided into 5 groups: low-dose TSU-68 monotherapy (100 mg/kg/day), low-/ high-dose paclitaxel monotherapy (10 and $20 \mathrm{mg} / \mathrm{kg} / \mathrm{day}$, respectively), low-dose combination therapy $(100 \mathrm{mg} / \mathrm{kg} /$ day of TSU-68 and $10 \mathrm{mg} / \mathrm{kg} /$ day of paclitaxel), and control (vehicle) groups.

Evaluation of treatment response. Subcutaneous tumor volume was calculated using the following formula: maximum diameter $\mathrm{x}$ minimum diameter ${ }^{2} \times 1 / 2$. The rate of subcutaneous tumor proliferation was calculated using the following formula: tumor volume on the day of measurement/tumor volume at the start of drug therapy.

Statistical analysis. Significance was tested using the unpaired Student's t-test. $\mathrm{P}<0.05$ was regarded as significant.

\section{Results}

Inhibitory effects of TSU-68 on subcutaneous tumor proliferation. In the high-dose TSU-68 monotherapy group, the rate of proliferation was significantly lower than in the control group ( $p<0.05$, Fig. 1), and the oral administration of TSU-68 alone inhibited endometrial tumor proliferation.

Inhibitory effects of low-dose combination therapy with TSU-68 and paclitaxel on subcutaneous tumor proliferation. There were no significant differences in the rate of proliferation between the low-dose TSU-68/paclitaxel monotherapy and

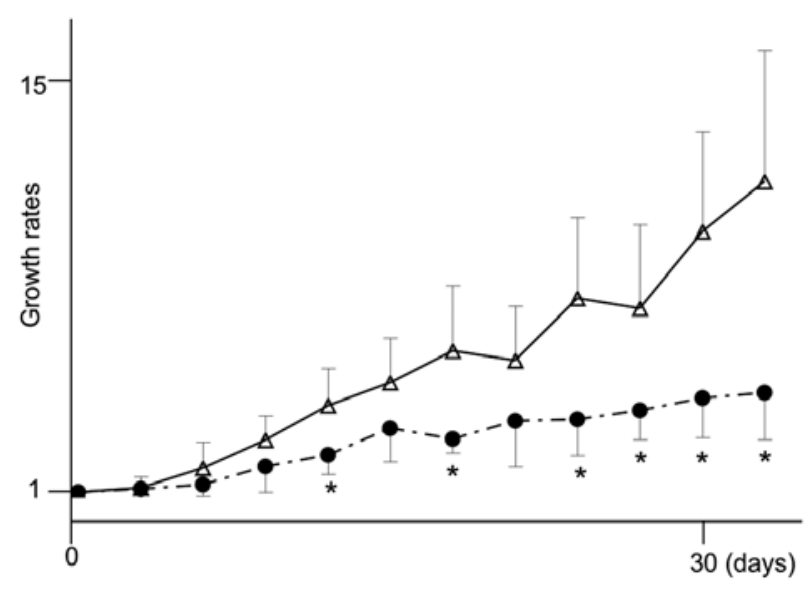

Figure 1. Effects of TSU-68 monotherapy on the subcutaneous tumor. From two weeks after the subcutaneous inoculation of the HEC1A cell strain of endometrial cancer $\left(6 \times 10^{6}\right)$, TSU-68 at $200 \mathrm{mg} / \mathrm{kg} /$ day (black circle, $\mathrm{n}=6$ ) or a vehicle (white triangle, $n=6$ ) were orally administered every day. The rate of tumor proliferation after the start of drug administration, which was significantly lower in the TSU-68-treated group than in the control (vehicle) group $\left({ }^{*} \mathrm{p}<0.05\right)$, is shown.

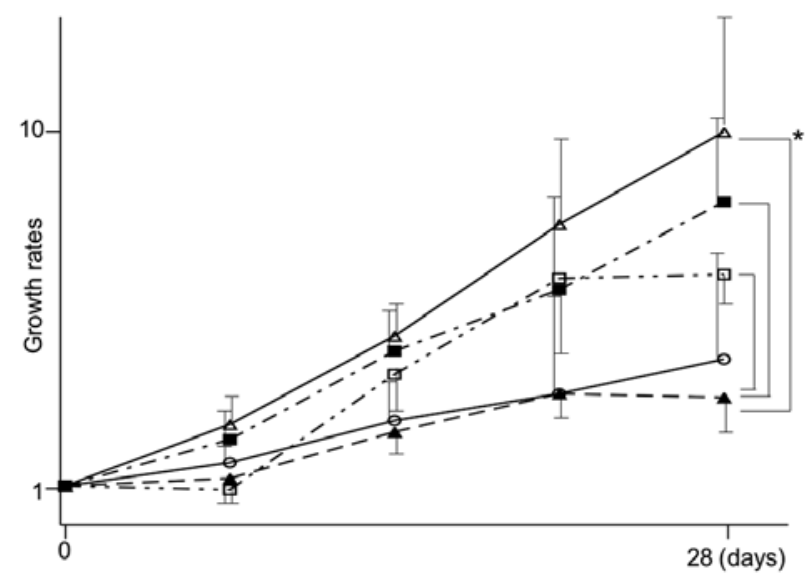

Figure 2. Effects of combination therapy with TSU-68 and paclitaxel on the subcutaneous tumor. From 2 weeks after the subcutaneous inoculation of HEC1A, TSU-68 was orally administered every day, and paclitaxel was intraperitoneally injected once a week. Based on treatment methods, animals were divided into 5 groups: a control (vehicle) group (white triangle, $n=12$ ), a group treated with $100 \mathrm{mg} / \mathrm{kg} /$ day of TSU-68 (black square, $\mathrm{n}=4$ ), a group treated with $10 \mathrm{mg} / \mathrm{kg} /$ day of paclitaxel (white square, $\mathrm{n}=4$ ), a group treated with $20 \mathrm{mg} / \mathrm{kg} /$ day of paclitaxel (white circle, $\mathrm{n}=8$ ), and a group treated with the combination of TSU-68 at $100 \mathrm{mg} / \mathrm{kg} / \mathrm{day}$ and paclitaxel at $10 \mathrm{mg} / \mathrm{kg} / \mathrm{day}$ (black triangle, $\mathrm{n}=4$ ). Neither single low-dose TSU-68 nor paclitaxel therapies significantly inhibited tumor proliferation in comparison with the control group. Low-dose combination therapy significantly inhibited tumor proliferation in comparison with vehicle treatment and respective single low-dose therapy $\left({ }^{*} \mathrm{p}<0.01\right)$.

control groups. In the low-dose combination therapy group, the rate of proliferation was significantly lower than in the control and respective low-dose monotherapy groups $(\mathrm{p}<0.01)$. In the high-dose paclitaxel monotherapy group, tumor proliferation was significantly inhibited in comparison with the control group $(\mathrm{p}<0.01)$. However, there was no significant difference between this and low-dose paclitaxel monotherapy groups. 


\section{Discussion}

In this study using a mouse model, the oral administration of TSU-68 alone inhibited the proliferation of subcutaneously implanted endometrial cancer. In addition, combination therapy with TSU-68 and paclitaxel at a low dose, one at which respective monotherapy was not effective, inhibited tumor proliferation.

Currently, some molecule-targeting agents are applied in clinical practice to treat various malignant tumors. In Europe and the United States, tyrosine kinase inhibitors such as imatinib mesilate, gefitinib, erlotinib, the Raf kinase inhibitor sorafenib, the anti-HER2 antibody trastuzumab and the anti-human VEGF monoclonal antibody bevasizumab, have been approved and applied in clinical practice to treat non-small cell lung, kidney, breast and metastatic rectal cancer, respectively $(18,19)$.

Few studies have reported molecule-targeting therapy for endometrial cancer. Kamat et al reported that expression of the most important angiogenic factor, VEGF, was marked in more than $50 \%$ of patients with endometrial cancer, suggesting that high-level VEGF expression is an independent prognostic factor (20). Based on their findings, we designed the current study, considering that VEGF-targeting therapy may be effective for endometrial cancer.

TSU-68 is a small-molecular-weight synthetic kinase inhibitor of the receptors Flk-1/KDR, PDGFR 3 and FGFR1 $(13,14)$. We previously reported the inhibitory and survivalprolonging effects of orally-administered TSU-68 on tumor angiogenesis and peritoneal dissemination using a peritoneallydisseminated ovarian cancer model in mice (21). The current study, also using a mouse model, demonstrated the antitumor effects of TSU-68 on subcutaneously implanted endometrial cancer, suggesting the usefulness of molecule-targeting therapy with TSU-68 for endometrial cancer. As the HEC1A endometrial cancer cell strain employed in this study secreted a large amount of VEGF (data not shown), the inhibitory effects of TSU-68 on tumor proliferation, as observed in this study, may have been mediated by VEGF.

Phase I clinical studies of TSU-68 in patients with solid tumors have been conducted $(22,23)$. Dose-limiting toxicities included abdominal pain, anorexia, nausea/vomiting, pericardial effusion and thrombocytopenia $(22,23)$. According to Kuenen et al, the maximum tolerated dose was $100 \mathrm{mg} / \mathrm{m}^{2}$ t.i.d., and no objective response was achieved in any patient. As a sufficient plasma level is not obtained at this dose, monotherapy with TSU-68 may not be effective (22).

Currently, the tumor-regressing effects of moleculetargeting therapy alone are limited. Therefore, in clinical practice, it is combined with conventional anticancer drugs in many patients (24-28). As a representative regimen, combination therapy with bevasizumab, fluorouracil, and leucovor for colorectal cancer is known $(27,28)$. TSU-68 may also be clinically useful when combined with anticancer drugs.

Garofalo et al conducted a basic study using a peritoneallydisseminated ovarian cancer mouse model to evaluate the effects of combination therapy with TSU-68 and paclitaxel (29). The combination of TSU-68 at $200 \mathrm{mg} / \mathrm{kg} / \mathrm{day}$ orally administered every day and paclitaxel at $20 \mathrm{mg} / \mathrm{kg} /$ day intravenously injected once a week achieved synergistic inhibitory effects on tumor proliferation/ascites production and survival-prolonging effects in comparison with monotherapy (29). Naumova et al performed a basic study to clarify the mechanism involved in the efficacy of combination therapy with TSU-68 and paclitaxel (30). The combination more potently inhibited the proliferation of human umbilical vein endothelial cells (HUVECs) and human microvascular endothelial cells, and induced HUVEC apoptosis compared to monotherapy (30).

Paclitaxel causes the stabilization/hyperplasia of microtubules by inhibiting their depolymerization, and exhibits anticancer actions by suppressing cell division (31). The agent also inhibits endothelial cell proliferation at a low concentration $(32,33)$, acting not only on tumors but also on blood vessels, which may contribute to the effects of combination therapy with TSU-68.

In a clinical study, the plasma levels of TSU-68 administered at a tolerated dose were low, and TSU-68 alone may not be effective. In this basic study, the combination of TSU-68 and paclitaxel at a low dose, at which respective monotherapy does not inhibit tumor proliferation, exhibited inhibitory effects on tumor proliferation. Combination therapy with these two agents may be a new strategy for the treatment of endometrial cancer.

\section{Acknowledgements}

We thank K. Yonekura and M. Fukushima (Taiho Pharmaceutical Co., Ltd., Tokyo, Japan) for helpful discussions. The skillful technical assistance of Mrs. M. Ohashi is appreciated.

\section{References}

1. Jemal A, Siegel R, Ward E, Murray T, Xu J and Thun MJ: Cancer statistics, 2007. CA Cancer J Clin 57: 43-66, 2007.

2. Wolfson AH, Sightler SE, Markoe AM, Schwade JG, Averette HE, Ganjei $P$ and Hilsenbeck SG: The prognostic significance of surgical staging for carcinoma of the endometrium. Gynecol Oncol 45: 142-146, 1992.

3. Van Wijk FH, Huikeshoven FJ, Abdulkadir L, Ewing PC and Burger CW: Stage III and IV endometrial cancer: a 20-year review of patients. Int Gynecol Cancer 16: 1648-1655, 2006.

4. Folkman J: Tumor angiogenesis. Adv Cancer Res 43: 175-203, 1985.

5. Ruegg $\mathrm{C}$ and Mutter N: Anti-angiogenic therapies in cancer: achievements and open questions. Bull Cancer 94: 753-762, 2007.

6. Ferrara N: Molecular and biological properties of vascular endothelial growth factor. J Mol Med 77: 527-543, 1999.

7. Hermansson M, Nister M, Betsholtz C, Heldin CH, Westermark B and Funa K: Endothelial cell hyperplasia in human glioblastoma: coexpression of mRNA for platelet-derived growth factor (PDGF) $\mathrm{B}$ chain and PDGF receptor suggests autocrine growth stimulation. Proc Natl Acad Sci USA 85: 7748-7752, 1988.

8. Williams B, Quinn-Baker A and Gallacher B: Serum and plateletderived growth factor-induced expression of vascular permeability factor mRNA by human vascular smooth muscle cells in vitro. Clin Sci 88: 141-147, 1995.

9. Seghezzi G, Patel S, Ren CJ, Gualandris A, Pintucci G, Robbins ES, Shapiro RL, Galloway AC, Rifkin DB and Mignatti P: Fibroblast growth factor-2 (FGF-2) induces vascular endothelial growth factor (VEGF) expression in the endothelial cells of forming capillaries: an autocrine mechanism contributing to angiogenesis. J Cell Biol 141: 1659-1673, 1998.

10. Sivridis E: Angiogenesis and endometrial cancer. Anticancer Res 21: 4383-4388, 2001

11. McMeekin DS, Sill MW, Benbrook D, Darcy KM, StearnsKurosawa DJ, Eaton L and Yamada SD: Gynecologic Oncology Group A phase II trial of thalidomide in patients with refractory endometrial cancer and correlation with angiogenesis biomarkers: a Gynecologic Oncology Group study. Gynecol Oncol 105: 508-516, 2007 
12. Giatromanolaki A, Sivridis E, Brekken R, Thorpe PE, Anastasiadis P, Gatter KC, Harris AL and Koukourakis MI: The angiogenic 'vascular endothelial growth factor/flk-1(KDR) receptor' pathway in patients with endometrial carcinoma: prognostic and therapeutic implications. Cancer 92: 2569-2577, 2001.

13. Laird AD, Vajkoczy P, Shawver LK, Thurnher A, Liang C, Mohammadi M, Schlessinger J, Ullrich A, Hubbard SR, Blake RA, Fong TA and Strawn LM: TSU-68 is a potent antiangiogenic and antitumor agent that induces regression of established tumors. Cancer Res 60: 4152-4160, 2000.

14. Hoekman K: TSU-68, a multitargeted angiogenesis inhibitor. Cancer 7: 134-138, 2001.

15. Laird AD, Christensen JG, Li G, Carver J, Smith K, Xin X, Moss KG, Louie SG, Mendel DB and Cherrington JM: TSU-68 inhibits Flk-1/KDR and PDGFRbeta in vivo, resulting in rapid apoptosis of tumor vasculature and tumor regression in mice. FASEB J 16: 681-690, 2002.

16. Scudder SA, Liu PY, Wilczynski SP, Smith HO, Jiang C, Hallum AV III, Smith GB, Hannigan EV, Markman M and Alberts DS: Paclitaxel and carboplatin with amifostine in advanced, recurrent, or refractory endometrial adenocarcinoma: a phase II study of the Southwest Oncology Group. Gynecol Oncol 96: 610-615, 2005.

17. Pectasides D, Pectasides E and Economopoulos T: Systemic therapy in metastatic or recurrent endometrial cancer. Cancer Treat Rev 33: 177-190, 2007.

18. Murdoch D and Sager J: Will targeted therapy hold its promise? An evidence-based review. Ann Surg Oncol 14: 942-953, 2007.

19. Steeghs N, Nortier JW and Gelderblom H: Small molecule tyrosine kinase inhibitors in the treatment of solid tumors: an update of recent developments. Ann Surg Oncol 14: 942-953, 2007.

20. Kamat AA, Merritt WM, Coffey D, Lin YG, Patel PR, Broaddus R, Nugent E, Han LY, Landen CN Jr, Spannuth WA, Lu C, Coleman RL, Gershenson DM and Sood AK: Clinical and biological significance of vascular endothelial growth factor in endometrial cancer. Clin Cancer Res 13: 7487-7495, 2007.

21. Machida S, Saga Y, Takei Y, Mizuno I, Takayama T, Kohno T, Konno R, Ohwada M and Suzuki M: Inhibition of peritoneal dissemination of ovarian cancer by tyrosine kinase receptor inhibitor SU6668 (TSU-68). Int J Cancer 114: 224-229, 2005.

22. Kuenen BC, Giaccone G, Ruijter R, Kok A, Schalkwijk C, Hoekman K and Pinedo HM: Dose-finding study of the multitargeted tyrosine kinase inhibitor SU6668 in patients with advanced malignancies. Clin Cancer Res 11: 6240-6246, 2005.
23. Sessa C, Viganò L, Grasselli G, Trigo J, Marimon I, Lladò A, Locatelli A, Ielmini N, Marsoni S and Gianni L: Phase I clinical and pharmacological evaluation of the multi-tyrosine kinase inhibitor SU006668 by chronic oral dosing. Eur J Cancer 42: 171-178, 2006.

24. Finley RS: Overview of targeted therapies for cancer. Am J Health Syst Pharm 60: 4-10, 2003.

25. Moses MA, Harper J and Fernandez CA: A role for anti-angiogenic therapy in breast cancer. Curr Oncol Rep 6: 42-48, 2004.

26. Margolin K, Gordon MS, Holmgren E, Gaudreault J, Novotny W, Fyfe G, Adelman D, Stalter S and Breed J: Phase Ib trial of intravenous recombinant humanized monoclonal antibody to vascular endothelial growth factor in combination with chemotherapy in patients with advanced cancer: pharmacologic and long-term safety data. J Clin Oncol 19: 851-856, 2001.

27. Hurwitz HI, Fehrenbacher L, Hainsworth JD, Heim W, Berlin J, Holmgren E, Hambleton J, Novotny WF and Kabbinavar F: Bevacizumab in combination with fluorouracil and leucovorin: an active regimen for first-line metastatic colorectal cancer. J Clin Oncol 23: 3502-3508, 2005.

28. Hurwitz H, Fehrenbacher L, Novotny W, Cartwright T, Hainsworth J, Heim W, Berlin J, Baron A, Griffing S, Holmgren E, Ferrara N, Fyfe G, Rogers B, Ross R and Kabbinavar F: Bevacizumab plus irinotecan, fluorouracil, and leucovorin for metastatic colorectal cancer. N Engl J Med 350: 2335-2342, 2004

29. Garofalo A, Naumova E, Manenti L, Ghilardi C, Ghisleni G, Caniatti M, Colombo T, Cherrington JM, Scanziani E, Nicoletti MI and Giavazzi R: The combination of the tyrosine kinase receptor inhibitor SU6668 with paclitaxel affects ascites formation and tumor spread in ovarian carcinoma xenografts growing orthotopically. Clin Cancer Res 9: 3476-3485, 2003.

30. Naumova E, Ubezio P, Garofalo A, Borsotti P, Cassis L, Riccardi E, Scanziani E, Eccles SA, Bani MR and Giavazzi R: The vascular targeting property of paclitaxel is enhanced by SU6668, a receptor tyrosine kinase inhibitor, causing apoptosis of endothelial cells and inhibition of angiogenesis. Clin Cancer Res 12: 1839-1849, 2006.

31. Spencer CM and Faulds D: Paclitaxel. A review of its pharmacodynamic and pharmacokinetic properties and therapeutic potential in the treatment of cancer. Drugs 48: 794-847, 1994.

32. Belotti D, Vergani V, Drudis T, Borsotti P, Pitelli MR, Viale G, Giavazzi $\mathrm{R}$ and Taraboletti G: The microtubule-affecting drug paclitaxel has antiangiogenic activity. Clin Cancer Res 2: $1843-1849,1996$.

33. Wang J, Lou P, Lesniewski R and Henkin J: Paclitaxel at ultra low concentrations inhibits angiogenesis without affecting cellular microtubule assembly. Anticancer Drugs 14: 13-19, 2003. 